\title{
Dust formation in oxygen-rich circumstellar shells around long-period variables
}

\author{
Kyung Sook Jeong, Jan Martin Winters, Erwin Sedlmayr \\ Institut für Astronomie und Astrophysik, Technische Universität Berlin, \\ Sekr. PN 8-1, Hardenbergstr. 36, D-10623 Berlin, Germany
}

\begin{abstract}
We present the first steps of our work aimed at a consistent time-dependent modeling of oxygen-rich circumstellar dust shells (CDS) around pulsating AGB stars. The nature of the most likely nucleation seeds is investigated for this situation and we find that $\mathrm{TiO}_{2}$ is a most promising candidate to serve as the primary condensate, forming already at temperatures well above $1000 \mathrm{~K}$. These nuclei evolve to macroscopic dust grains by heterogeneous growth processes involving several chemical species. We investigate the varying chemical composition of the resulting dust grains as they evolve as a function of time in a fluid element moving through the CDS of an oxygen-rich long-period variable star (LPV).
\end{abstract}

\section{Introduction}

Circumstellar shells around cool pulsating late type stars are the major sources for the replenishment of the interstellar medium with processed material, in particular in the form of complex molecules and of solid dust particles (e.g. Gehrz 1989). Contrary to the carbon-rich situation, for which consistent dust shell models have been presented e.g. by Fleischer et al. (1992) and by Feuchtinger et al. (1993), a consistent physical description of CDS around oxygen-rich LPVs is still lacking. This is mainly due to the more complex problem of inorganic dust formation in an oxygen-rich environment. In contrast to the carbonrich situation, where mainly homogeneous amorphous carbon grains and also PAHs are formed, in the case of an oxygen-rich composition of the gas almost all carbon is locked up in the chemically inert $\mathrm{CO}$ molecule. Therefore, grains only can be formed from molecules containing the remaining oxygen and those abundant heavy elements $(\mathrm{Fe}, \mathrm{Si}, \mathrm{Mg}, \mathrm{S}, \mathrm{Al}$ and $\mathrm{Ti})$, which are contained in reactive gas phase species. In this case a heterogeneous composition of the emerging dust component has to be expected.

\section{Basic considerations}

In order to account for the observed high optical depths of the dust shells, formation of the major dust components requires the contribution of relatively abundant elements. Circumstellar dust is often observed at temperatures around $1000 \mathrm{~K}$ or even higher. Therefore, the dust grains must consist of (a mixture of) high temperature condensates. Fig. 1 depicts the most promis- 

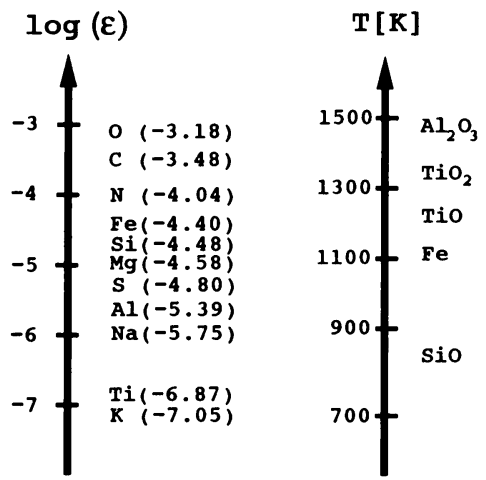

Figure 1. Solar element abundances and stability hierarchy of high temperature condensates (see text)

ing candidates for dust formation resulting from these criteria. The temperatures refer to a supersaturation ratio $S=1$ for a mass density of $\rho=10^{-12} \mathrm{~g} \mathrm{~cm}^{-3}$. Corundum becomes stable around $1500 \mathrm{~K}$, titanium oxides around $1300 \mathrm{~K}$, iron around $1100 \mathrm{~K}$, and silicon monoxide around $900 \mathrm{~K}$. However, this stability hierarchy does not necessarily mean that the respective compounds are actually formed in the circumstellar environment.

\section{Nucleation seeds}

Basically, the formation of dust grains can be conceived as a two step process: i) formation of a critical cluster which serves as a condensation seed and ii) growth of these clusters to macroscopic dust grains by addition of suitable molecules. Step one, the formation of the critical cluster requires high supersaturation ratios $(S \gg 1)$, whereas the growth process, i.e. condensation of the growth molecules on preexisting nucleation seeds proceeds already if $S>1$ for the respective growth species.

In order to determine the most likely primary condensation seed, nucleation rates $J_{*}$ were calculated for the candidates shown in Fig. 1 in a range of the $p-T$ plane, relevant for the inner region of a dust forming circumstellar shell. Contour plots of the resulting nucleation rates are shown in Fig. 2 for various seed nuclei, which were calculated from kinetic nucleation theory (see Patzer et al. 1998b) assuming chemical equilibrium in the gas phase. This assumption of chemical equilibrium may be considered as highly unrealistic in pulsating situations, but due to the large numerical expenditure, no consistent non-equilibrium chemical structure exists so far for Miras and LPVs.

Since $\mathrm{Al}_{2} \mathrm{O}_{3}$ is already stable at very high temperatures, it might be expected to be the first condensate in the cooling outflows of oxygen-rich stars (e.g. Kozasa \& Sogawa, this volume). However, using the thermodynamic functions resulting from quantum mechanical $a b$ initio calculations for the structure of the $\mathrm{Al}_{2} \mathrm{O}_{3}$ molecule to calculate its equilibrium concentration shows that this molecule is virtually not present in the gas phase (see Chang et al. 1998, Patzer 


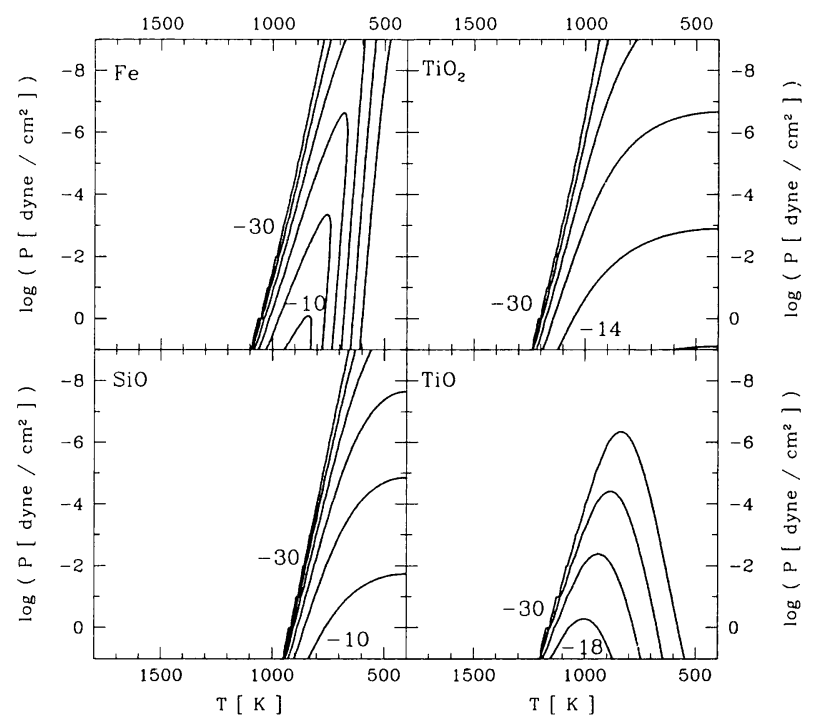

Figure 2. Contour lines of the nucleation rate for different nucleating species in the $\mathrm{p}-\mathrm{T}$ plane. Contour levels are plotted in steps of $\Delta \log \left(J_{*} / n_{<\mathrm{H}>}\right)=4$.

et al. 1998a).Therefore, homogeneous nucleation of molecular $\mathrm{Al}_{2} \mathrm{O}_{3}$ from the gas phase seems to be very unlikely in a circumstellar environment. However, the analysis of meteorites reveals that corundum-bearing grains in fact are formed in the circumstellar shells of oxygen-rich AGB stars (e.g. Zinner 1998). The formation of solid $\mathrm{Al}_{2} \mathrm{O}_{3}$ in principle might proceed via chemical reactions like $\mathrm{Al}_{2} \mathrm{O}+2 \mathrm{H}_{2} \mathrm{O} \rightarrow \mathrm{Al}_{2} \mathrm{O}_{3}+2 \mathrm{H}_{2} \mathrm{O}$ (e.g. Gail \& Sedlmayr 1998) or $2 \mathrm{Al}+3 \mathrm{H}_{2} \mathrm{O} \rightarrow$ $\mathrm{Al}_{2} \mathrm{O}_{3}+3 \mathrm{H}_{2}$ (Kozasa \& Hasegawa 1987) five body collision, respectively. Such a scheme seems to be realistic for the formation of corundum in a grain mantle, where the reaction partner can be adsorbed at the grain surface for a certain time. For the formation of an isolated $\left(\mathrm{Al}_{2} \mathrm{O}_{3}\right)_{N}$ cluster by this kind of chemical reactions, at least $N$ (simultaneous) three body collisions in the gas phase are required, the effective occurrence of which is a delicate question considering the involved time scales. In the following we therefore consider corundum only as a growth species and are left with $\mathrm{SiO}, \mathrm{Fe}, \mathrm{TiO}$ and $\mathrm{TiO}_{2}$ for the nucleation process (see Fig. 2).

Although the $\mathrm{SiO}$ molecule is the most abundant candidate for nucleation in oxygen-rich circumstellar shells, its nucleation rate only peaks around $600 \mathrm{~K}$, which is significantly lower than the typical temperatures at the inner edge of the dust shells as derived e.g. from infrared interferometry (Danchi et al. 1994). Fe nucleation occurs only at temperatures well below $1000 \mathrm{~K}$. Only the titanium oxides show relevant nucleation rates at temperatures above $1000 \mathrm{~K}$ where $\mathrm{TiO}_{2}$ nucleation is by far more efficient, due to its higher molecular concentration in the relevant region of the $p-T$ plane. 

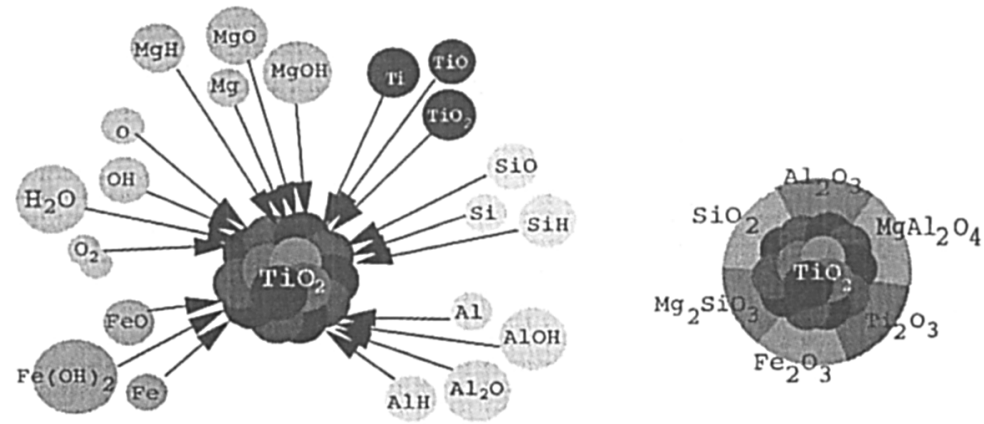

Figure 3. Heterogeneous growth on the seed material $\mathrm{TiO}_{2}$.

l.h.s.: molecules contributing to grain growth; r.h.s.: sketch of some compounds constituting the resulting grain mantle

\section{Heterogeneous growth on $\mathrm{TiO}_{2}$ grain nuclei}

The $\mathrm{TiO}_{2}$ seed nuclei grow by accretion of those molecular species which can form a stable condensate under the thermodynamic conditions prevailing in the circumstellar environment. The respective monomer of these condensates does not necessarily have to exist in the gas phase, but may be formed by chemical reactions on the grain surface from the atoms and molecules present in the gas phase (see Fig. 3).

For example, $\mathrm{Mg}_{2} \mathrm{SiO}_{4}$ can be formed by addition of the atoms $\mathrm{Mg}, \mathrm{Si}$ and $\mathrm{O}$, and the molecules $\mathrm{O}_{2}, \mathrm{MgO}, \mathrm{SiO}, \mathrm{SiO}_{2}$, etc. Actual formation of such a solid compound requires that all the generalized supersaturation ratios of the contributing atomic and molecular species are larger than unity (Dominik et al. 1993). Their method is applied here to describe this heterogeneous growth process. Generally, several compounds are simultaneously stable at a given pressure and temperature and there is a hierarchy of dust materials which successively become stable with decreasing temperature. The first condensing compounds are aluminum-bearing species like $\mathrm{Al}_{6} \mathrm{Si}_{2} \mathrm{O}_{13}, \mathrm{Al}_{2} \mathrm{SiO}_{5}$ and $\mathrm{Al}_{2} \mathrm{O}_{3}$, followed by $\mathrm{Mg}_{2} \mathrm{SiO}_{4}, \mathrm{MgSiO}_{3}, \mathrm{SiO}_{2}$ and by the iron oxides and $\mathrm{MgO}$, while sulphur-bearing compounds like $\mathrm{MgSO}_{4}$ and $\mathrm{SiS}_{2}$ only become stable at temperatures around $600 \mathrm{~K}$. This stability hierarchy (shown in Fig. 4) might depend on the total gas pressure (which affects the partial pressure of the contributing species in a nonlinear way) as can be seen e.g. from the stability limit of $\mathrm{MgAl}_{2} \mathrm{O}_{4}$ depicted in Fig. 4 (see also Gail \& Sedlmayr 1998).

\section{First results of a consistent model calculation}

The consistent description of a dust forming circumstellar shell constitutes a non-linearly coupled problem comprising hydrodynamics, thermodynamics, chemistry, dust formation, growth and evaporation and the radiative transfer problem. Therefore, a simultaneous solution of the complete problem describing the dust forming system is necessary for a reliable description of a circumstellar dust shell. Applying the numerical method described in Fleischer et al. (1992), we 


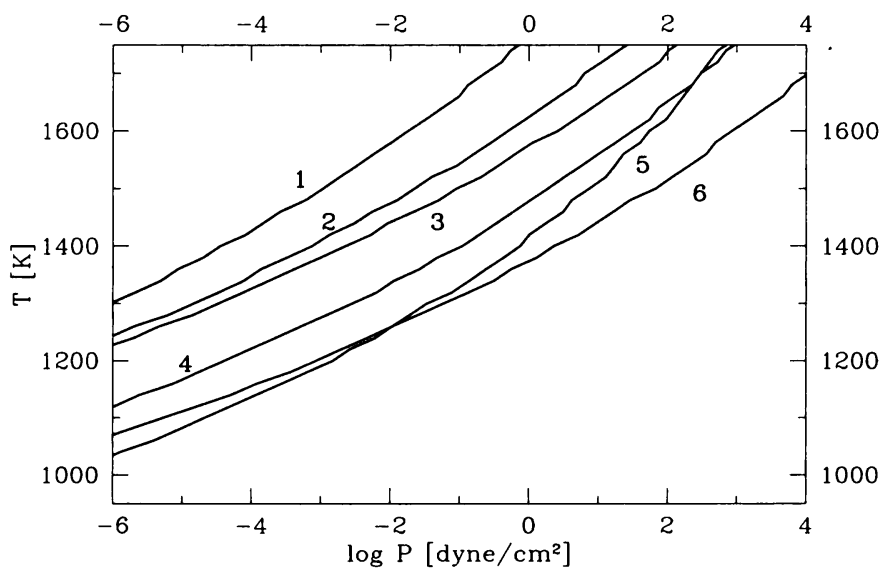

Figure 4. Stability limits $(S=1)$ for some high temperature condensates in the p-T plane. 1: $\mathrm{Al}_{2} \mathrm{O}_{3}, 2: \mathrm{Al}_{6} \mathrm{Si}_{2} \mathrm{O}_{13}, 3: \mathrm{Ti}_{2} \mathrm{O}_{3}, 4: \mathrm{MgSiO}_{3}$, 5: $\mathrm{MgAl}_{2} \mathrm{O}_{4}, 6: \mathrm{MgTiO}_{3}$

calculate a consistent dust shell model including a proper treatment of heterogeneous dust formation as outlined in the preceding sections. The solution of the equation system is completely determined by prescribing the values of 6 parameters for which we choose $\mathrm{L}_{*}=2 \cdot 10^{4} \mathrm{~L}_{\odot}, \mathrm{T}_{*}=2000 \mathrm{~K}, \mathrm{M}_{*}=1 \mathrm{M}_{\odot}$, velocity amplitude at the inner boundary $\Delta \mathrm{u}_{p}=5 \mathrm{kms}^{-1}, \mathrm{P}=300$ days, $\epsilon_{C} / \epsilon_{O}=0.5$, and solar abundances for the other elements. This model produces a mass loss rate of $\dot{M} \approx 5 \cdot 10^{-5} \mathrm{M}_{\odot} \mathrm{yr}^{-1}$ and an outflow velocity of $v_{\infty} \approx 15 \mathrm{~km} \mathrm{~s}^{-1}$. The radii of most of the dust grains range between 0.01 and $0.1 \mu \mathrm{m}$, but there still exist a few grains larger than $0.5 \mu \mathrm{m}$. Fig. 5 displays the temperature and velocity structure of the dust shell model at a fixed instant of time and a sketch of the chemical composition of the dust grains at different locations in the atmosphere. At $\mathrm{R} \approx 1.9 \mathrm{R}_{*}$ the temperature is around $1200 \mathrm{~K}$ and most of the dust volume consists of $\mathrm{Al}_{6} \mathrm{Si}_{2} \mathrm{O}_{13}$. Titanium-bearing compounds like $\mathrm{MgTiO}_{3}$ and titanium oxides like $\mathrm{Ti}_{2} \mathrm{O}_{3}$ and $\mathrm{Ti}_{3} \mathrm{O}_{5}$ are also included just after the onset of condensation, but their volume fraction is almost negligible. Already at slightly lower temperatures $\left(\mathrm{T} \approx 1100 \mathrm{~K}\right.$ at $\mathrm{R} \approx 2.2 \mathrm{R}_{*}$ ), $\mathrm{Mg}_{2} \mathrm{SiO}_{4}, \mathrm{MgSiO}_{3}$ and $\mathrm{SiO}_{2}$ are the dominant compounds contained in the dust grains. Just below $1000 \mathrm{~K}$ the iron oxides are formed, which finally contribute about $30 \%$ to the dust volume. All sulphur-bearing compounds become only stable around $600 \mathrm{~K}$ and their volume fraction remains well below $10 \%$.

This investigation about the composition of dust grains, together with the laboratory work (e.g. Jäger et al. 1994, Koike et al. 1993), will enable us to get a more precise insight about the "astrophysical silicates" which are considered as being responsible not only for the well known amorphous silicate bands, but also for several emission features recently detected at wavelengths between 12 and $70 \mu \mathrm{m}$ (e.g. Waters \& Molster, this volume).

Acknowledgments. This work has been supported by the FAZIT Stiftung and by the BMBF (grant 05 3BT13A 6). 


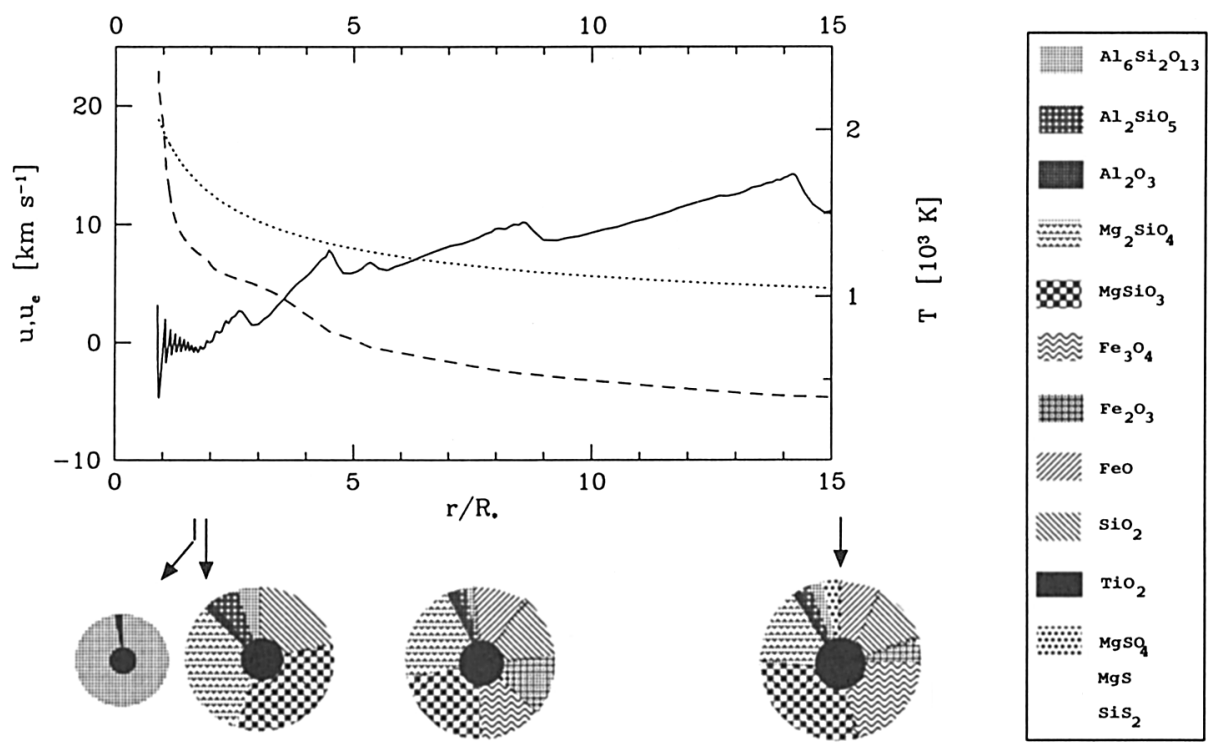

Figure 5. Upper panel: velocity (solid line), escape velocity (dotted line), and temperature structure (dashed line) of the dust shell model. Lower panel: composition of the dust component at different distances from the star.

\section{References}

Chang C., Patzer A.B.C., Sedlmayr E., Sülzle D., 1998, Eur. Phys. J. D 2, 57

Danchi W.C., Bester M., Degiacomi C.G., Greenhill L.J., Townes C.H., 1994, AJ 107, 1469

Dominik C., Sedlmayr E., Gail H.-P., 1993, A\&A 277, 578

Feuchtinger M.U., Dorfi E.A., Höfner S., 1993, A\&A 273, 513

Fleischer A.J., Gauger A., Sedlmayr E., 1992, A\&A 266, 321

Gail H.-P., Sedlmayr E., 1998, in Chemistry and physics of molecules and grains in space, Faraday Discussion no. 109, P. Sarre (ed.), London, p. 303

Gehrz R.D., 1989, in Interstellar Dust, L.J. Allamandola and A.G.G.M. Tielens (eds.), Kluwer Academic Publishers, Dordrecht, p. 445

Jäger C., Mutschke H., Begemann B., Dorschner J., Henning T., 1994, A\&A 292,641

Koike C., Shibai H., Tuchiyama A., 1993, MNRAS 264, 654

Kozasa T., Hasegawa H., 1987, Prog. Theor. Phys 77, 1402

Patzer A.B.C., Chang C., Sedlmayr E., Sülzle D., 1998a, Eur. Phys. J. D, submitted

Patzer A.B.C., Gauger A., Sedlmayr E., 1998b, A\&A 337, 847

Zinner E., 1998, Ann. Rev. Earth Planet. Sci 26, 147 